\title{
Descriptif de l'activité des structures des urgences en Île-de-France à l'aide du questionnaire « National Emergency Department Inventory » (NEDI) appliqué au système français
}

\author{
Characteristics of Emergency Departments in the Paris Metropolitan Area: \\ the NEDI-Paris Survey
}

\author{
N. Lemachatti $\cdot$ R. Hellmann $\cdot$ M. Wargon $\cdot$ P. Hausfater $\cdot$ B. Riou $\cdot$ A.F. Sullivan $\cdot$ C.A. Camargo $\cdot$ Y. Freund \\ Reçu le 30 novembre 2017; accepté le 24 janvier 2018 \\ (C) SFMU et Lavoisier SAS 2018
}

Résumé Introduction : La base de données NEDI (National Emergency Department Inventory) repose sur un descriptif de l'activité des structures des urgences (SU). Elle rend compte de la diversité de ces structures au niveau national et international. L'objectif de cette étude NEDI-Paris était de décrire l'organisation des SU en Île-de-France et les moyens à disposition, selon le statut public ou privé.

Méthode : Les SU ouvertes 24 heures/24 et 7 jours/7 ont été inclus. Un questionnaire comprenant 29 items a permis de recueillir les caractéristiques de ces services et les données relatives à l'activité de l'année 2015. Un taux de participation de $80 \%$ était souhaité dans le cadre des enquêtes NEDI pour assurer une exhaustivité des résultats.

Résultats : Sur 117 services éligibles, 97 ont participé à

\author{
N. Lemachatti $(\bowtie) \cdot$ P. Hausfater $\cdot$ B. Riou $\cdot$ Y. Freund \\ Sorbonne université, Paris, France \\ e-mail : lemnajla@gmail.com \\ N. Lemachatti \\ Service d'accueil des urgences, hôpital Tenon, \\ Assistance publique-Hôpitaux de Paris (AP-HP), F-75020 Paris, \\ France \\ R. Hellmann \\ Service d'accueil des urgences, \\ hôpital Bichat, AP-HP, F-75018 Paris, France \\ M. Wargon \\ Service Urgences SMUR, \\ Centre hospitalier de Saint-Denis, \\ Rue du Dr Delafontaine, F-93200 Saint-Denis, France \\ P. Hausfater $\cdot$ B. Riou $\cdot$ Y. Freund \\ Service d'accueil des urgences, \\ hôpital Pitié-Salpêtrière, AP-HP, F-75013 Paris, France \\ A.F. Sullivan · C.A. Camargo \\ Service d'accueil des urgences, \\ Massachusetts General Hospital, Boston, États-Unis
}

l'étude, soit un taux de réponse de $83 \%$. L'ensemble de ces services était localisé dans des établissements hospitaliers. Le service était sur un seul site géographique dans $86 \%$ des cas. Le nombre annuel médian de visites était de 34000 (interquartile : $23000-56000$ ). Soixante-six centres $(68 \%)$ appartenaient au système public, dont 22 centres universitaires et 31 centres (32\%) étaient des services privés. Les urgences adultes, pédiatriques et mixtes représentaient respectivement 54, 26 et $20 \%$ de l'offre de soins. Un temps d'attente inférieur à une heure était rapporté par $25 \%$ des services : $42 \%$ pour les services privés versus $17 \%$ dans le public $(p=0,01)$. Un taux d'hospitalisation inférieur à $20 \%$ était rapporté par $54 \%$ des centres, similaire entre public et privé ( 60 vs $50 \% ; p=0,40)$. Parmi les services interrogés, $22 \%$ ont déclaré fonctionner en surcharge, plus fréquemment dans le public comparativement aux services privés (27 vs $10 \%$ ). La gestion 24 heures/24 et 7 jours/7 de certaines pathologies de même que la disponibilité de certains spécialistes étaient le plus souvent observées dans les hôpitaux publics, notamment universitaires.

Conclusion: L'enquête NEDI en Île-de-France a montré une hétérogénéité dans les caractéristiques et les moyens à disposition des SU. Un déterminant majeur de ces disparités repose sur le statut public versus privé. Une réflexion peut être menée à partir de ces résultats.

Mots lés : Médecine d'urgence · Île-de-France · Public · Privé · Universitaire

Abstract Aims: The National Emergency Department
Inventory (NEDI) project is comprised of several local and
national surveys of emergency departments (EDs) in a spe-
cific region. These databases reflect the diversity of EDs
worldwide. The purpose of our study was to describe the
characteristics of EDs in Paris (France) metropolitan area,
with particular attention to available resources and capacity 
of these EDs depending on their public or private status. Procedure: All EDs opened 24/7 were included. The previously-used NEDI survey included 29 items regarding services characteristics during the year 2015. A response rate of at least $80 \%$ was required for study success.

Results: Out of the 117 eligible EDs, 97 participated (83\% response rate). All of these EDs were located in hospitals. The vast majority of EDs (86\%) had a contiguous layout of care. The median annual ED visit rate was 34,000 (interquartile range, 23,000-56,000). Sixty-six centers were public $(68 \%)$ including 22 academic centers, and $31(32 \%)$ were private (non-academic). EDs caring for adults only, children only, and both represented $54 \%, 26 \%$, and $20 \%$ of our sample, respectively. They reported a median waiting time of less than one hour in $25 \%$ of cases: $42 \%$ for private EDs vs $17 \%$ for public EDs $(P=0.01)$. There was $54 \%$ of centers that reported a mean admission rate of below $20 \%$, similar between private and public sectors $(60 \%$ vs $50 \%$, respectively; $P=0.40$ ). Surveyed centers reportedly suffered from running overcapacity in $22 \%$ of cases that was more frequent in public EDs ( $27 \%$ vs $10 \%$ in private EDs). Public hospitals presented with available 24/7 management of diverse pathologies as well as specialist referral more often than in private centers, and particularly in academic centers.

Conclusion: The NEDI-Paris survey showed significant variability in the characteristics and resources of Paris area EDs. A major driver of these differences appeared to be whether the ED was in public or private sector. Our survey establishes a benchmark to reflect on improvements in Paris area emergency care.

Keywords International emergency medicine $\cdot$ Paris area France $\cdot$ Public $\cdot$ Private institution $\cdot$ Academic status

\section{Introduction}

Les structures des urgences (SU) occupent une place essentielle dans la réponse aux besoins de soins non programmés. Ils constituent un mode d'entrée de plus en plus fréquent dans le système de soins [1]. En France, depuis leur création au milieu des années 1960, les SU ont connu une croissance soutenue de leur activité. Le nombre de passages a augmenté de $64 \%$ entre 1990 et 2001, soit une progression annuelle de $4,6 \%$ [2]. La fréquentation s'est encore accrue de près de $30 \%$ depuis cette date pour atteindre 18,4 millions de passages en 2012 [3]. De façon similaire, les SU en Îlede-France enregistraient en 2014 près de trois millions de passages [4]. Face à cette forte demande, plusieurs réformes ont permis de faire progressivement émerger et structurer cette activité hospitalière [5]. La publication de décrets sur l'organisation et l'accueil des SU, la seniorisation de la prise en charge, l'intégration des temps de garde au temps de travail et la réduction de ce dernier, la mise en œuvre de « plans nationaux d'urgence » avec la création de postes d'urgentistes, le développement de circuits courts ont ainsi profondément modifié l'organisation et les moyens des SU en France. Plus récemment, la création d'un diplôme d'études spécialisées a consacré la reconnaissance de la médecine d'urgence comme discipline à part entière [6].

En France, comme dans tous des pays dotés de structure des urgences, l'amélioration de la qualité des soins dispensés et du fonctionnement de ces services repose sur une meilleure compréhension des déterminants liés à la demande et à 1 'offre $[7,8]$. Afin de répondre à cet objectif et de mieux caractériser les SU dans le monde, la base de données internationale NEDI (National Emergency Departement Inventory) a été créée à l'initiative de l'Emergency Medicine Network (EmNet) » à Boston (États-Unis) [9]. Elle s'appuie sur plusieurs enquêtes menées dans différents pays [10-16]. L'élaboration d'un panorama de l'activité des urgences a ainsi permis de développer cette base commune visant à s'enrichir de l'expérience d'autres systèmes de soins [17]. Les différents professionnels de santé peuvent s'appuyer sur celle-ci pour appréhender le fonctionnement des urgences et proposer des pistes de réflexion sur l'accès aux soins non programmés. En Europe, les enquêtes NEDI ont été menées au Danemark, en Slovénie et en Suisse [13,14,16]. L'objectif principal de notre étude était de réaliser un descriptif de l'activité des urgences en Île-de-France sur le même modèle que les précédentes études NEDI, pouvant ainsi servir d'élément de comparaison avec d'autres structures.

\section{Matériel et méthodes}

Il s'agissait d'une étude descriptive conduite entre janvier et avril 2016. Selon la définition donnée par NEDI États-Unis, un service de médecine d'urgence correspondait à un service pouvant dispenser des soins 24 heures/24 et 7 jours/7 [9]. Une liste de l'ensemble des départements privés et publics de la région parisienne a été obtenue auprès de la Haute Autorité de santé (HAS). Tous les services éligibles ont été contactés par téléphone afin de mettre à jour les coordonnées. Les particularités spécifiques à l'organisation du système hospitalier français ont été prises en compte : hôpitaux publics, structures privées, services à but lucratif, centres hospitaliers universitaires (CHU). La supervision de l'étude était menée conjointement avec l'EmNet.

Un questionnaire comprenant 29 items a été adressé par courrier électronique aux responsables d'unité des centres participants. La trame de ce questionnaire était fondée sur le questionnaire envoyé aux SU des précédentes études NEDI [18]. Il portait spécifiquement sur l'activité de l'année 2015. Les questions reposaient sur plusieurs 
thématiques : caractéristiques générales des $\mathrm{SU}$, prise en charge et devenir du patient, moyens matériels et humains à disposition. Un taux de réponse d'au moins $80 \%$ était attendu, en accord avec les consignes du projet NEDI [17].

\section{Analyse statistique}

Les variables continues étaient exprimées en moyenne ( \pm écart-type [ET]) ou médiane (intervalle interquartile). Les variables catégorielles étaient rapportées en nombre et en pourcentage. Les caractéristiques des services d'accueil des urgences des hôpitaux publics et privés ont été comparées. Nous avons également réalisé une comparaison entre les $\mathrm{SU}$ accueillant respectivement plus et moins de 50000 patients par an. Les pourcentages ont été comparés à l'aide du test de Wald Chi-Square ou du test de Fisher. Toutes les comparaisons statistiques ont été effectuées en bilatérale, avec un risque alpha de 0,05 . Les analyses statistiques ont été réalisées avec le logiciel NCSS 12.0 (Statistical Solution, Cork, Irlande).

\section{Résultats}

Nous avons dénombré 117 services de médecine d'urgence dont 97 ont accepté de participer à l'étude, soit un taux de participation de $83 \%$. La population d'étude comptait $21 \mathrm{CHU}(22 \%), 45$ centres hospitaliers publics non universitaires $(46 \%)$ et 31 services SU de structures privées (32\%). La répartition des centres n'ayant pas répondu était la suivante : 7 services privés et 13 centres publics non universitaires.

La part des urgences adultes et pédiatriques représentait respectivement 54 et $26 \%$. Seuls $20 \%$ des services accueillaient une population mixte (adultes et enfants). Certains SU mixtes (65\%), appartenant aux centres hospitaliers publics, présentaient la particularité d'accueillir des urgences pédiatriques uniquement traumatologiques. L'ensemble des services interrogés était fondé dans des établissements hospitaliers, dont $68 \%$ appartenaient au service public. Le taux annuel médian de visite des centres participants était de 34000 (interquartile : 23 000-56 000) (Tableau 1) : 51000 pour les $\mathrm{CHU}$ versus 34500 pour les centres publics non universitaires et 24000 pour les structures privées ( $p=$ $0,001)$ (Tableau 2). La majorité des SU (86\%) se situait sur un seul site géographique.

Dans 58 SU (60\%), moins de $20 \%$ des patients arrivaient aux urgences par ambulance. Un temps d'attente moyen inférieur à une heure était rapporté dans $25 \%$ des centres, avec une différence significative entre établissements privés et publics (respectivement 42 vs $17 \% ; p=0,01$ ) (Tableau 2). Dans 52 SU (54\%), moins de $20 \%$ des patients faisaient l'objet d'une hospitalisation après avoir été vus aux urgences (Tableau 1). Le taux d'admission était similaire entre CHU, centres publics non universitaires et centres privés (Tableau 2). Parmi les participants, $22 \%$ ont estimé que leur service fonctionnait en surcharge : $27 \%$ des centres publics versus $10 \%$ des centres privés ( $p=0,04)$ et $29 \%$ étaient des SU pédiatriques. La taille du service ne semblait pas associée à un risque accru de surcharge (Tableau 2).

Un médecin était physiquement présent 24 heures/24 et 7 jours $/ 7$ dans 95 services (98\%). Quatre-vingt-sept départements $(90 \%)$ bénéficiaient de la présence continue d'un staff infirmier. Un scanner exclusivement dédié aux urgences n'était disponible que pour $24 \%$ des services. L'accès à l'imagerie n'était pas significativement différent entre CHU, centres publics non universitaires et établissements privés (respectivement 33, 27 et 13\%) (Tableau 2). Environ $70 \%$ des participants ont déclaré avoir un laboratoire dédié aux urgences 24 heures $/ 24$ et 7 jours/ 7 : CHU, hôpitaux publics non universitaires et centres privés, respectivement 76,71 et $61 \%$ (Tableau 2). Un défibrillateur et un respirateur étaient immédiatement disponibles, respectivement dans 97 et $95 \%$ des services interrogés (Tableau 1). Concernant les pathologies pouvant être traitées 24 heures/24 et 7 jours/7, la prise en charge des urgences dentaires, ophtalmologiques et ORL ne concernaient qu'une faible proportion des centres participants. Ces derniers étaient en majorité des établissements publics : $100 \%$ pour les urgences dentaires, $63 \%$ pour les urgences ophtalmologiques et $73 \%$ pour les urgences ORL. La gestion continue des urgences psychiatriques n'était également assurée que dans $24 \%$ des services interrogés, dont $91 \%$ relevaient du service public (Tableau 3 ).

\section{Discussion}

Notre étude portait sur un inventaire des caractéristiques des SU en Île-de-France. Il en ressortait une hétérogénéité dans l'organisation et les ressources de ces structures. Les différences observées semblaient résider essentiellement dans le statut public versus privé. Notre enquête recensait près de $70 \%$ d'établissements publics. Bien que concentrant un grand nombre de structures d'urgences, l'offre de soins en région parisienne est globalement superposable au dispositif national, la France comptant $75 \%$ de SU appartenant au système public [3]. Une répartition similaire de l'offre de soins était observée dans l'étude NEDI menée en Suisse et à Singapour [14,15]. À l'inverse, plus de la moitié des SU de Bogota (Colombie) appartient au système privé [12]. En Îlede-France, la part des patients pris en charge dans le privé reste moindre comparativement au système public : $14 \%$ des passages sans hospitalisation et $10 \%$ des passages suivis d'hospitalisation en 2014 [4]. Dans notre enquête, 
Tableau 1 Caractéristiques des structures d'urgence en Île-de-France IIQ : intervalle interquartile

\begin{tabular}{|c|c|}
\hline Variable & Valeur \\
\hline & $n=97$ \\
\hline Service d'accueil des urgences adultes & $49(51 \%)$ \\
\hline Service d'accueil des urgences pédiatrique & $24(25 \%)$ \\
\hline Services mixtes (adultes + enfants) & $24(25 \%)$ \\
\hline Service localisé dans un hôpital ou une clinique & $97(100 \%)$ \\
\hline Département de médecine d'urgence indépendant & $66(68 \%)$ \\
\hline Disposition contiguë & $84(88 \%)$ \\
\hline Patients redirigés à l'accueil & $38(39 \%)$ \\
\hline Hôpitaux publics & $66(68 \%)$ \\
\hline Dont centres hospitaliers universitaires & $22(22 \%)$ \\
\hline Centre privé : appartenant au service public & $11(11 \%)$ \\
\hline Centre privé : à but lucratif & $21(22 \%)$ \\
\hline Taux annuel de visites & $34000\left[\begin{array}{llll}23 & 000-56 & 000\end{array}\right]$ \\
\hline Nombre de lits hospitaliers & $380[220-560]$ \\
\hline Nombre de box d'examen & $10[6-16]$ \\
\hline \multicolumn{2}{|l|}{ Prise en charge et devenir des patients } \\
\hline \multicolumn{2}{|l|}{$\%$ de patients arrivant aux urgences par ambulance } \\
\hline$<20 \%$ & $59(60 \%)$ \\
\hline $20-39 \%$ & $30(31 \%)$ \\
\hline $40-59 \%$ & $8(8 \%)$ \\
\hline $60 \%$ & $1(1 \%)$ \\
\hline \multicolumn{2}{|l|}{ Temps moyen d'attente } \\
\hline$<1$ heure & $24(25 \%)$ \\
\hline$>1$ heure & $73(75 \%)$ \\
\hline \multicolumn{2}{|l|}{$\%$ d'hospitalisation après passage aux urgences } \\
\hline$<20 \%$ & $52(54 \%)$ \\
\hline $20-39 \%$ & $40(41 \%)$ \\
\hline $40-59 \%$ & $4(4 \%)$ \\
\hline $60 \%$ & $1(1 \%)$ \\
\hline \multicolumn{2}{|l|}{ Moyens matériels et humains } \\
\hline Urgentiste présent sur site $24 / 7$ & $95(98 \%)$ \\
\hline Staff infirmier sur site $24 / 7$ & $87(90 \%)$ \\
\hline Scanner dédié aux urgences & $23(24 \%)$ \\
\hline Défibrillateur & $94(97 \%)$ \\
\hline Respirateur & $92(95 \%)$ \\
\hline Chambre à pression négative & $1(1 \%)$ \\
\hline Logiciel de recueil de données cliniques & $92(95 \%)$ \\
\hline Accès à Internet & $97(100 \%)$ \\
\hline
\end{tabular}

l'opposition entre public et privé reposait sur trois éléments : temps d'attente, surcharge, moyens humains et techniques.

Un quart des services interrogés a rapporté avoir des temps d'attente inférieurs à une heure, dont plus de la moitié était des services privés. Ce résultat laisserait suggérer qu'une prise en charge plus rapide serait majoritairement le fait des établissements privés et que ces derniers seraient plus performants que les hôpitaux publics. Plusieurs hypothèses pourraient expliquer les différences observées : struc- tures plus petites, moins de passages notamment la nuit, sélection des patients à l'admission, impératifs de rentabilité et de productivité propres aux services privés, patients moins graves et de fait moins chronophages. Ces résultats avaient déjà été avancés dans une enquête menée en 2014 dans les SU d'Île-de-France. L'étude objectivait une médiane de passage de 92 min dans le privé versus 168 min dans le public [4]. Les données relatives aux temps d'attente et de passage sont cependant à considérer avec précaution, d'autant plus 
Tableau 2 Comparaison des structures d'urgence des centres hospitaliers publics universitaires, publics non universitaires et privés en Île-de-France et services accueillant $>50000$ et $<50000$ passages par an

\begin{tabular}{|c|c|c|c|c|c|c|c|}
\hline Variable & Universitaire & $\begin{array}{l}\text { Non } \\
\text { universitaire }\end{array}$ & $\begin{array}{l}\text { Privé } \\
\text { Non } \\
\text { universitaire }\end{array}$ & $\begin{array}{l}\text { Valeur } \\
\text { de } P^{*}\end{array}$ & $\begin{array}{l}\text { Taux } \\
\text { de passages } \\
\text { Annuel < } \\
50000\end{array}$ & $\begin{array}{l}\text { Taux } \\
\text { de passages } \\
\text { Annuel > } \\
50000\end{array}$ & $\begin{array}{l}\text { Valeur } \\
\text { de } P \S\end{array}$ \\
\hline & $n=21$ & $n=45$ & $n=31$ & & $n=65$ & $n=32$ & \\
\hline Taux annuel (médiane) & 51000 & 34500 & 24000 & $<0,001$ & & & \\
\hline $\begin{array}{l}\text { Nombre de lits } \\
\text { hospitaliers (médiane) }\end{array}$ & 600 & 450 & 220 & $<0,001$ & 524 & 250 & $<0,001$ \\
\hline $\begin{array}{l}\text { Nombre de box } \\
\text { d'examen (médiane) }\end{array}$ & 16 & 11 & 7 & $<0,001$ & 16 & 8 & $<0,001$ \\
\hline $\begin{array}{l}\text { Temps moyen d'attente } \\
<1 \mathrm{~h}\end{array}$ & $6(29 \%)$ & $5(11 \%)$ & $13(42 \%)$ & 0,01 & $18(28 \%)$ & $6(18 \%)$ & 0,33 \\
\hline $\begin{array}{l}\text { Taux moyen } \\
\text { d'hospitalisation }< \\
20 \%\end{array}$ & $9(43 \%)$ & $23(55 \%)$ & $18(60 \%)$ & 0,4 & $34(56 \%)$ & $16(50 \%)$ & 0,66 \\
\hline Surcharge & $4(19 \%)$ & $14(32 \%)$ & $3(10 \%)$ & 0,004 & $13(20 \%)$ & $8(25 \%)$ & 0,61 \\
\hline $\begin{array}{l}\text { Scanner dédié } \\
\text { aux urgences }\end{array}$ & $7(33 \%)$ & $12(27 \%)$ & $4(13 \%)$ & 0,09 & $12(19 \%)$ & $11(33 \%)$ & 0,13 \\
\hline Laboratoire $24 / 7$ & $16(76 \%)$ & $32(71 \%)$ & $19(61 \%)$ & 0,26 & $41(64 \%)$ & $26(79 \%)$ & 0,17 \\
\hline
\end{tabular}

que ces indicateurs restent largement utilisés par les institutions aussi bien publiques que privées pour communiquer sur leurs prises en charge et ne rendent pas forcément compte de la qualité des soins dispensés, des moyens à disposition ou encore des caractéristiques des patients. Une étude nationale datant de 2013 avait montré que l'accueil et l'orientation des patients aux urgences étaient réalisés dans la demiheure qui suivait l'arrivée pour neuf patients sur dix et le début effectif des soins dans l'heure pour sept patients sur dix. Néanmoins, il était constaté que les patients âgés ou encore ceux admis pour un motif relevant de la traumatologie attendaient plus longtemps le début des soins [19]. Des résultats similaires avaient été retrouvés dans une étude française : les sujets âgés étaient les plus à risque d'être pris en charge en dehors des délais attribués par le système de triage propre aux urgences [20].

Dans notre enquête, 22 \% des services rapportaient avoir fonctionné en surcharge pour l'année 2015. Il s'agissait majoritairement d'établissements publics. On notait que près de $40 \%$ de ces services étaient des services pédiatriques. Les urgences pédiatriques représentaient en 2014 près de $30 \%$ des trois millions de passages recensés en Île-de-France [4]. Une étude datant de 2008 avait établi un état des lieux des urgences pédiatriques en France. Il en ressortait une insuffisance de locaux et d'effectifs en termes de personnel médical [21]. L'activité pédiatrique présente également la particularité en France d'être surtout visible au moment des épidé- mies hivernales. Face à cet afflux saisonnier, le nombre de passages aux urgences peut considérablement augmenter, et le nombre d'hospitalisations peut excéder les capacités d'aval [22]. L'ensemble de ces éléments peut expliquer la proportion de services pédiatriques s'étant déclarés en surcharge pour l'année passée. De façon générale, le phénomène de surcharge des SU est international, objectivé dans plusieurs études NEDI. Près de $50 \%$ des SU suisses et colombiennes et $40 \%$ des SU chinoises ont déclaré fonctionner à flux tendu $[11,12,14]$. Plusieurs stratégies ont été implémentées, visant à réduire la surcharge des SU et à faire face à l'afflux massif. On peut notamment citer l'exemple des fast track systems ou circuits courts, dont l'efficacité dans le management du flux aux urgences a été mise en évidence [23]. En France, ils sont implantés dans environ un tiers des SU et accueillent près de $30 \%$ des patients [3].

Les disparités concernant la prise en charge de certaines pathologies et la disponibilité des spécialistes aux urgences peuvent aussi être discutées à l'issue de notre enquête. On peut citer l'exemple de la cardiologie. Seule la moitié des centres participants a déclaré pouvoir bénéficier d'un avis cardiologique 24 heures/ 24 et 7 jours/ 7 . Les pathologies cardiovasculaires occupent cependant une place importante dans le recours aux soins ; elles constituent la deuxième cause de décès en France. En 2008, en Île-de-France, les séjours pour affections de l'appareil cardiovasculaire représentaient $10 \%$ du total des séjours hospitaliers [24]. Une 
Tableau 3 Pourcentage des structures d'urgence en Île-de-France pouvant prendre en charge différentes pathologies 24 heures/ 24 et 7 jours $/ 7$

\begin{tabular}{|lll|}
\hline Spécialités & Pathologies (exemples) & PEC 24/7 (\%) \\
\hline Cardiologie & Arythmie, infarctus du myocarde & 57 \\
Oncologie & Neutropénie fébrile & 83 \\
Médicales (autres) & Infection urinaire, crise d'asthme & 97 \\
Traumatologie & Accident de la voie publique & 84 \\
Neurologie et neurochirurgie & Accident vasculaire cérébral, hémorragie méningée & 18 \\
Obstétrique & Éclampsie & 23 \\
Gynécologie & Rupture de kyste ovarien, salpingite & 63 \\
Otorhinolaryngologie & Épistaxis sévère & 15 \\
Ophtalmologie & Glaucome aigu & 8 \\
Toxicologie & Overdose, intoxication au monoxyde de carbone & 40 \\
Psychiatrique & Décompensation psychotique & 24 \\
Dentaire & Alluvion dentaire & 5 \\
Chirurgie maxillofaciale & Fracture mandibulaire, cellulite & 8 \\
Chirurgie plastique & Plaies faciales sévères & 16 \\
Chirurgie de la main & Atteinte tendineuse & 10 \\
Orthopédie & Facture du col du fémur & 72 \\
Chirurgie viscérale & Appendicite aiguë & 81 \\
\hline PEC : prise en charge & & \\
\hline
\end{tabular}

enquête menée en 2010 en Île-de-France dans les unités de soins intensifs cardiologiques avait conclu que les ressources en personnel médical étaient insuffisantes, ne permettant pas d'assurer la permanence des soins. De même que les problématiques d'aval en termes de lits compromettaient la viabilité à long terme de certaines unités de cardiologie [25]. L'action des soins intensifs de cardiologie s'intégrant dans une filière de soins, leurs insuffisances de fonctionnement peuvent alors se répercuter sur la qualité des soins dispensés aux urgences. Au même titre que la cardiologie, la disponibilité des psychiatres dans les SU en Île-de-France pose question. Seul un quart des services interrogés pouvaient bénéficier de l'accès à un avis psychiatrique H24. Une étude datant de 2003 pointait les insuffisances de la prise en charge des urgences psychiatriques au sein des SU parisiennes. Les insuffisances concernaient un défaut de personnel infirmier qualifié et de locaux adaptés aux patients psychiatriques [26]. L'étude NEDI menée à Pékin (Chine) rapportait une situation plus préoccupante avec une couverture quasi inexistante des urgences psychiatriques, à l'inverse des services de Singapour pouvant accueillir à $80 \%$ ce type d'urgences $[11,15]$.

Notre étude présente plusieurs limites. Elle repose sur des données déclaratives, induisant un probable biais dans les réponses. Néanmoins, la comparaison des données de notre étude avec les résultats d'un audit mené par l'Agence régionale de santé (ARS) n'objective pas d'incohérence [4]. Plusieurs centres ont refusé de participer. Les données manquantes ont pu là aussi induire un biais, au même titre que ces centres peuvent différer de notre population d'étude. Toutefois, l'objectif de $80 \%$ de réponse a été atteint. En second lieu, le questionnaire NEDI n'est pas un outil validé. À notre connaissance, il n'existe cependant pas un tel instrument permettant d'évaluer des SU à travers le monde. On pourra cependant souligner que le recours à ce questionnaire a été éprouvé avec succès dans plusieurs études internationales NEDI, confirmant ainsi son adaptabilité à différents systèmes de médecine d'urgence $[9,18]$. Autres limites pouvant être relevées : les centres participants fournissaient des données approximatives lorsque les données chiffrées exactes étaient manquantes. Des notions telles que « surcharge » n'avaient pas été préalablement définies, mais laissées à la libre appréciation des services interrogés. L'étude suggère des différences entre public et privé. On ne peut toutefois pas conclure que les différences observées sont uniquement imputables au statut des SU. D'une part, la méthodologie de l'enquête NEDI ne permet pas d'établir un lien de causalité. D'autre part, d'autres caractéristiques propres à ces services peuvent constituer des facteurs confondants et expliquer les disparités. Enfin, l'impact sur la qualité des soins des différences observées entre public et privé n'était pas rapporté à l'issue de l'enquête et pourrait faire l'objet d'une étude supplémentaire.

\section{Conclusion}

Notre étude pose les bases d'une description régionale de l'offre de soins d'urgence en Île-de-France et met en 
évidence certaines disparités entre SU publiques et privées. Cette hétérogénéité comprend en particulier les temps d'attente, la surcharge ou l'accès aux plateaux techniques. Cette enquête NEDI peut constituer une base de réflexion pour la gestion et répartition de l'offre de soins dans les SU en Îlede-France.

Liens d'intérêts Les auteurs déclarent ne pas avoir de liens d'intérêts.

\section{Références}

1. Schuur JD, Venkatesh A (2012) The growing role of emergency departments in hospital admissions. N Engl J Med 367:391-3

2. Direction de l'hospitalisation et de l'organisation des soins (2003) Circulaire DHOS/O $1 \mathrm{n}^{\mathrm{o}}$ 2003-195 du 16 avril 2003 relative à la prise en charge des urgences. http://circulaire.legifrance.gouv.fr/ index.php?action $=$ afficherCirculaire $\&$ hit $=1 \& \mathrm{r}=15832$ (Dernier accès le 30 novembre 2017)

3. Cour des comptes (2014) Rapport sur l'application des lois de financement de la sécurité sociale. Les urgences hospitalières: une fréquentation croissante, une articulation avec la médecine de ville à repenser. https://www.ccomptes.fr/fr/publications/securite-sociale-2014 (Dernier accès le 30 novembre 2017)

4. Commission régionale d'experts urgences Île-de-France (2016) Activité des services d'urgences Île-de-France. Année 2015. https:// www.iledefrance.ars.sante.fr/sites/default/files/2017-02/UrgencesCREU-Rapport-IDF-2015.pdf (Dernier accès le 30 novembre 2017)

5. République française (2006) Décret $n^{\circ}$ 2006-577 du 22 mai 2006 relatif à la médecine d'urgence et modifiant le code de la santé publique. J.O.R.F. no 119 du 22 mai 2006. https://www.legifrance. gouv.fr/affichTexte.do?cidTexte=JORFTEXT000000788652\&categorieLien=id (Dernier accès le 30 novembre 2017)

6. République française (2015) Journal officiel de la République française. Ministère de l'Éducation nationale, de l'Enseignement supérieur et de la Recherche. Arrêté du 13 novembre 2015 fixant la liste des diplômes d'études spécialisées de médecine. JORF $\mathrm{n}^{\circ}$ $0281 \mathrm{du} 4$ décembre 2015. https://www.legifrance.gouv.fr/affichTexte.do?cidTexte=JORFTEXT000031560595 (Dernier accès le 30 novembre 2017)

7. Yiadom MY, Ward MJ, Chang AM, et al (2015) Consensus statement on advancing research in emergency department operations and its impact on patient care. Acad Emerg Med 22:757-64

8. Arnold JL (1999) International emergency medicine and the recent development of emergency medicine worldwide Ann Emerg Med 33:97-103

9. Sullivan AF, Richman IB, Ahn CJ, et al (2006) A profile of US emergency departments in 2001. Ann Emerg Med 48:694-701
10. Wen LS, Oshiomogho JI, Eluwa GI, et al (2012) Characteristics and capabilities of emergency departments in Abuja, Nigeria. Emerg Med J 29:798-801

11. Wen LS, Xu J, Steptoe AP, et al (2013) Emergency department characteristics and capabilities in Beijing, China. J Emerg Med 44:1174-79

12. Bustos Y, Castro J, Wen LS, et al (2015) Emergency department characteristics and capabilities in Bogotá, Colombia Int. J Emerg Med 8:30

13. Jaklic B, Wen LS, Sullivan AF, Camargo CA (2012) A profile of emergency departments in Slovenia ISRN Emerg Med 2012:6

14. Sanchez B, Hirzel AH, Bingisser R, et al (2013) State of emergency medicine in Switzerland: a national profile of emergency departments in 2006. Int J Emerg Med 6:23

15. Wen LS, Venkataraman A, Sullivan AF, Camargo CA (2012) National inventory of emergency departments in Singapore. Int J Emerg Med 5:38

16. Wen LS, Anderson PD, Stagelund S, et al (2013) National survey of emergency departments in Denmark. Eur J Emerg Med 20:205-9

17. Steptoe AP, Corel B, Sullivan AF, Camargo CA (2011) Characterizing emergency departments to improve understanding of emergency care systems Int J Emerg Med 4:42

18. Emergency Medicine Network (EMNet) (2017) The National ED inventories survey instrument. Available at: www.emnet-nedi.org. (Dernier accès le 30 novembre 2017)

19. Boisguérin B, Brilhault G, Ricroch L, et al (2015) « Premiers résultats de l'enquête nationale sur les structures d'urgences hospitalières réalisées par la DREES le 11 juin 2013 » dans Le Panorama des établissements de santé - édition 2014, DREES

20. Freund Y, Vincent-Cassy C, Bloom B, et al (2013) Association between age older than 75 years and exceeded target waiting times in the emergency department: a multicenter cross-sectional survey in the Paris metropolitan area, France. Ann Emerg Med 62:449-56

21. Hue V, Dubos F, Pruvost I, Martinot A (2011) Organisation et moyens de l'accueil des urgences pédiatriques : enquête nationale française en 2008. Arch Pédiatrie 18:142-8

22. Mercier JC, Belletre X, Lejay E (2015) Urgences pédiatriques : l'exemple de la gestion des épidémies hivernales. Rev Prat Monogr 65:47-50

23. Sanchez M, Smally AJ, Grant RJ, Jacobs LM (2016) Effects of a fast-track area on emergency department performance. J Emerg Med 31:117-20

24. Lecarpentier Y (2008) Prise en charge des maladies cardiaques. Haut Comité de santé publique (HCSP). Actualités et dossiers en santé publique $\mathrm{n}^{\circ} 63$. https://www.hcsp.fr/explore.cgi/Adsp? clef $=103$ (Dernier accès le 30 novembre 2017)

25. Agence régionale de santé (ARS) (2011) Dossier de presse. Organisation de l'offre de soins en cardiologie interventionnelle. www. ars.iledefrance.sante.fr (Dernier accès le 30 novembre 2017)

26. Dantchev N, Baubet T, Bigot T, et al (2003) Les urgences psychiatriques de l'AP-HP : urgences psychiatriques. Perspect Psychiatr 42:39-45 